\title{
Homozygous MED25 mutation implicated in eye-intellectual disability syndrome
}

\author{
Lina Basel-Vanagaite ${ }^{1,2,3,5} \cdot$ Pola Smirin-Yosef ${ }^{4,5} \cdot$ Jenna Lee Essakow $^{2} \cdot$ Shay Tzur $^{8}$. \\ Irina Lagovsky ${ }^{2,5} \cdot$ Idit Maya $^{3} \cdot$ Metsada Pasmanik-Chor $^{6} \cdot$ Adva Yeheskel $^{6}$. \\ Osnat Konen $^{2,7}$ • Naama Orenstein ${ }^{1,3}$ - Monika Weisz Hubshman ${ }^{1,2,3}$. \\ Valerie Drasinover ${ }^{3} \cdot$ Nurit Magal $^{3} \cdot$ Gaby Peretz Amit $^{3} \cdot$ Yael Zalzstein ${ }^{3}$. \\ Avraham Zeharia ${ }^{2,9} \cdot$ Mordechai Shohat $^{2,3,5}$ - Rachel Straussberg ${ }^{11,1,2}$. \\ Didier Monté $^{10} \cdot$ Mali Salmon-Divon ${ }^{4} \cdot$ Doron M. Behar $^{3,8}$
}

Received: 20 December 2014 / Accepted: 11 March 2015 / Published online: 20 March 2015

(C) Springer-Verlag Berlin Heidelberg 2015

\begin{abstract}
Genetic syndromes involving both brain and eye abnormalities are numerous and include syndromes such as Warburg micro syndrome, Kaufman oculocerebrofacial syndrome, Cerebro-oculo-facio-skeletal syndrome, Kahrizi syndrome and others. Using exome sequencing, we have been able to identify homozygous mutation p.(Tyr39Cys) in MED25 as the cause of a syndrome characterized by eye, brain, cardiac and palatal abnormalities as well as growth retardation, microcephaly and severe intellectual disability in seven patients from four unrelated families, all originating from the same village. The protein encoded by MED25 belongs to Mediator complex or MED complex, which is an evolutionary conserved multi-subunit
\end{abstract}

L. Basel-Vanagaite and P. Smirin-Yosef are equally contributed and shares first joint authorship.

Electronic supplementary material The online version of this article (doi:10.1007/s00439-015-1541-x) contains supplementary material, which is available to authorized users.

Lina Basel-Vanagaite

basel@post.tau.ac.il

1 Pediatric Genetics Unit, Schneider Children's Medical Center of Israel, Petach Tikva, Israel

2 Sackler Faculty of Medicine, Tel Aviv University, Tel Aviv, Israel

3 Raphael Recanati Genetics Institute, Rabin Medical Center, Beilinson Campus, Petach Tikva, Israel

4 Genomic Bioinformatics Laboratory, Department of Molecular Biology, Ariel University, Ariel, Israel

5 Felsenstein Medical Research Center, Rabin Medical Center, Petach Tikva, Israel

6 Bioinformatics Unit, G.S.W. Faculty of Life Sciences, Tel Aviv University, Tel Aviv, Israel
RNA polymerase II transcriptional regulator complex. The MED25 point mutation is located in the von Willebrand factor type A (MED25 VWA) domain which is responsible for MED25 recruitment into the Mediator complex; co-immunoprecipitation experiment demonstrated that this mutation dramatically impairs MED25 interaction with the Mediator complex in mammalian cells.

\section{Introduction}

The application of classical and high-throughput genomic technologies has led to the identification of a large number of genes involved in the pathogenesis of syndromes involving intellectual disability accompanied by eye and brain abnormalities. Examples of syndromes associated with both intellectual disability and congenital eye abnormalities include Warburg micro syndrome (MIM 600118, 614222, 615663, 614225), Kaufman oculocerebrofacial syndrome (MIM 244450), Cerebro-oculo-facio-skeletal syndrome

7 Imaging Department, Schneider Children's Medical Center of Israel, Petach Tikva, Israel

8 Molecular Medicine Laboratory, Rambam Health Care Campus, Haifa, Israel

9 Pediatric Day Hospitalization Department, Schneider Children's Medical Center of Israel, Petach Tikva, Israel

10 IRI USR 3078 CNRS, Parc CNRS de la Haute Borne, 50 avenue de Halley, B.P. 70478, 59658 Villeneuve d'Ascq Cedex, France

11 Department of Pediatric Neurology and Epilepsy Center, Schneider Children's Medical Center of Israel, Petach Tikva, Israel 
(COFS) (MIM 214150), Kahrizi syndrome (MIM 612713) and other syndromes. Molecular causes of many syndromes involving both eye and brain abnormalities still remain elusive. Autosomal recessive diseases are common in the Arab population of Israel, mostly as a result of the high rate of consanguinity. According to the previous studies (Jaber et al. 1994, 2000), up to $44 \%$ of marriages among Israeli Arabs were conducted between relatives, approximately half of which were between first cousins, although these numbers are becoming lower in recent years (Jaber et al. 1994, 2000; Basel-Vanagaite et al. 2006a).

We have identified seven individuals from four unrelated families living in the same village who were all affected by autosomal recessive intellectual disability. The disorder involves a wide range of symptoms including eye abnormalities, palatal abnormalities, thin corpus callosum, intellectual disability and other abnormalities. We report on the identification of homozygous MED25 mutation in all the affected individuals.

\section{Materials and methods}

\section{Subjects}

Subjects were enrolled in the study after a parental written informed consent was obtained. Additional informed consent was obtained from all individual participants for whom identifying information is included in this article. DNA samples were collected from 4 affected and 7 non-affected individuals belonging to 4 families.

\section{Whole exome sequencing}

In two of the families, exome sequencing was carried out; one affected individual and two control individuals from each family were examined (Family 1: I-1, I-2, II-10, Family 2: I-2, II-1, II-3).

Genomic DNA was extracted from peripheral leukocytes following standard protocols. Exome enrichment was achieved by the Nextera Rapid Capture Expanded Exome Kit (FC-140-1006) following manufacturer's guidelines. Sequencing was performed using the Illumina HiSeq 2500 machinery. The analytic pipeline available at the Genetics and Genomic Medicine Laboratory (Gene by Gene, Houston, Texas) was used to map the obtained fragments to the human reference genome (hg19), to generate the final list of candidate variants and to identify the variants of potential effect on function. Common variants in populations and variants without clear functional effect (intronic variants deeper than $\pm 10 \mathrm{bp}$, intergenic and synonymous variants) were usually filtered. The search for the variant(s) with potential causative role in the described phenotype was performed based on autosomal recessive inheritance mode and the familial segregation of the variants. We focused on private and rare (minor allele frequency $<0.01$ ) most likely damaging variants (splicing, missense, nonsense, truncating, frameshift) which were common to all the patients. While consanguinity between these two families was not reported, it is noteworthy that as both families reside in the same village which is known for high prevalence of inbreeding (Jaber et al. 1994, 2000); therefore, an autosomal recessive mode of inheritance was anticipated.

\section{Mutation detection by restriction analysis}

For detection of MED25 gene variant missense mutation c.116A $>$ G p.(Tyr39Cys) in exon 1 amplification of a 555bp fragment from genomic DNA was performed using the following primers: $5^{\prime}$-tgccetttctgagtcttcca- $3^{\prime}$ (forward), and $5^{\prime}$-ctttctccctccctcagacc- $3^{\prime}$ (reverse), for 36 cycles at an annealing temperature of $60{ }^{\circ} \mathrm{C}$. The mutation generates a restriction site, which digests the 555-bp fragment into 133 , 182 and 240-bp fragments. The fragment was digested with BTSI restriction enzyme (New England BioLabs), and the reaction products were separated by electrophoresis on $2 \%$ agarose gel.

\section{Bioinformatics methods}

MED25 was characterized using the UCSC Genome Browser [February 2009 (GRCh 37/hg 19)], NCBI [Build37.1 (August 2009)], and Ensembl Genome Browser [February 2009 (GRCh 37)]. Evolutionary conserved regions (ECR) browser analysis was performed on human genome (hg 19) to show MED25 gene pairwise alignments between human and various species, as follows: zebrafish (Danio rario, danRer7), rat (Rattus norvegicus, rn4), mouse (Mus musculus, mm10), and rhesus monkey (Rhesus macaque, rheMac3). Protein domains were delineated on MED25 homologs from 5 organisms mentioned above, using databases Interpro (Hunter et al. 2012), Pfam (Finn et al. 2014) and SUPERFAMILY (Wilson et al. 2009). Servers SIFT (Kumar et al. 2009) and PolyPhen-2 (Adzhubei et al. 2010) were used to predict mutation severity. A model structure for the von Willebrand factor type A domain (residues 14-225) of human MED25 was predicted using FFAS server (Jaroszewski et al. 2005), based on PDB file $1 \mathrm{~N} 3 \mathrm{Y}$, a von Willebrand factor type A domain of Integrin alpha-X/beta-2, as a template structure (Vorup-Jensen et al. 2003). The template shares $11 \%$ identity with the target. The model was built using Modeller program (Šali et al. 1995) and validated using model evaluation tools WHAT-IF (Vriend 1990), ModFold (McGuffin and Roche 2010) QMEAN (Benkert et al. 2008, 2009) and ProSA (Wiederstein and Sippl 2007). A sequence alignment of six 
different MED25 sequences from mammals was built using Uniprot server [Activities at the Universal Protein Resource (UniProt) 2014] and Mafft alignment program (Katoh and Standley 2013), then an HMMsearch (Finn et al. 2011) was performed to collect distant homologous sequences. A multiple sequence alignment of 102 sequences was built using Mafft (Katoh and Standley 2013). The alignment was projected on the 3D model structure, using ConSurf server (Landau et al. 2005). The Kyte-Doolittle hydrophobicity scale (Kyte and Doolittle 1982) was used for detecting hydrophobic regions.

\section{Plasmid construction, transfection and co-immunoprecipitation}

The human pCDNA3-Flag-NLS-MED25-Y39C mutant was generated by PCR using the previously described human pCDNA3-Flag-NLS-MED25 as a template (Verger et al. 2013) (primer sequences and detailed procedures are available upon request). 293-HEK cells were cultured in DMEM supplemented with $10 \%$ FCS (Gibco BRL). $6 \times 10^{6}$ cells were plated in $100 \mathrm{~mm}$ tissue culture dishes and transfected with $1 \mu \mathrm{g}$ of MED25 expression vectors using the Lipofectamine 2000 procedure (Lifetechnologies) for $18 \mathrm{~h}$. Transfected cells were lysed in lysis buffer (50 mM Tris/ $\mathrm{HCl}, \mathrm{pH} 8,250 \mathrm{mM} \mathrm{NaCl}, 1 \mathrm{mM}$ EDTA, $1 \mathrm{mM}$ TCEP, $10 \%$ glycerol, $3 \mathrm{mM} \mathrm{MgCl} 2,0.1 \%$ Igepal CA-630). After centrifugation, extracted proteins were immunoprecipitated overnight with anti-FLAG M2 magnetic beads (Thermo) at $4{ }^{\circ} \mathrm{C}$. After extensive washing, the fished proteins were detected by Western blot. The antibodies used were from Sigma (anti-Flag M2), from Santa-cruz (anti-Med6 and anti-Cdk8) or from Bethyl laboratories (anti-Med1, antiMed14, anti-Med16, anti-Med23 and anti-Med24).

\section{Real-time PCR population screening}

Screening for MED25 gene variant c.116A $>$ G [p. (Tyr39Cys), NM_030973] in random 80 healthy individuals was performed by polymerase chain reaction (PCR) with preamplification using gene-specific oligonucleotide primers (Supplemental Table S1), targeting MED25 gene, and a polymerase with proofreading activity. The samples were processed using automation to identify the zygosity at the nucleotide sites responsible for imidazolinone tolerance by RT-PCR for identification of a single-nucleotide polymorphism (SNP) using minor groove binder (MGB) type probes-Black Hole Quencher dye (BHQ-plus, Biosearch Technologies, Inc, Novato, CA, USA). To detect a MED25 gene mutation, probes were designed to differentiate the two alleles (Supplemental Table S1), and each probe was bound to a distinct fluorescent molecule that was detected using a duplex PCR. The two probes were specifically designed for hybridization to the non-mutant allele and to the mutant allele, respectively. The forward and reverse primers for amplification of the MED25 gene were mixed in the same well with the probes (mutant and non-mutant), generating fluorescent signals with different wavelengths. The emissions of the signal generated by the two probes were captured in real time by an Applied Biosystems 7900HT thermal cycler. The genotype of mutation sample was determined by comparison to a standard control of known genotype.

\section{Results}

\section{Clinical evaluation}

Clinical features that are shared by the affected individuals include intellectual disability, dysmorphic features, structural eye abnormalities, central nervous system abnormalities and nevus flammeus simplex on the forehead (Table 1).

Patient 1 (Fig. 2a, Family 1, II-5). This boy is the first affected child in the family. He died at the age of 8 years. Clinical features included congenital cataract and hypospadias. Additional features, as collected from his medical records, are presented in Table 1.

Patient 2 (Fig. 2a, Family 1, II-7) is the second affected child in family 1 . He died shortly after birth; no clinical records are available.

Patient 3 (Fig. 2a, Family 1, II-10) is the tenth of eleven children born to allegedly non-related Israeli Arab parents. Patient was born after an uneventful pregnancy at 40 weeks of gestation by vaginal delivery. Clinical features included congenital cataract and cleft palate; additional clinical features are listed in Table 1. At the age of 2 years, he was observed to be constantly smiling, presented with jerky movements, severe developmental delay and psychomotor retardation. Patient was still unable to crawl or speak but now had the ability to stand. During a visit at age three, the doctor once again observed severe psychomotor retardation. The individual demonstrated minimal movements, as well as a lack of concentration and eye contact. Brain MRI images of the affected individual at the age of 2 years and 8 months show abnormal hyperintense signal in the occipital periventricular white matter bilaterally (Fig. 1b).

Patient 4 (Fig. 2a, Family 2, II-2) is a sibling of Patient 5. She was born after an uneventful pregnancy at 40 weeks of gestation by vaginal delivery. At 16 months she was noted to be able to sit with some assistance but unable to stand. Severe developmental delay was noted. At the age of six, the individual was noted to have bilateral Achilles contractures and pes cavus. Clinical features included bilateral microcornea and cleft palate (additional features listed in Table 1). 


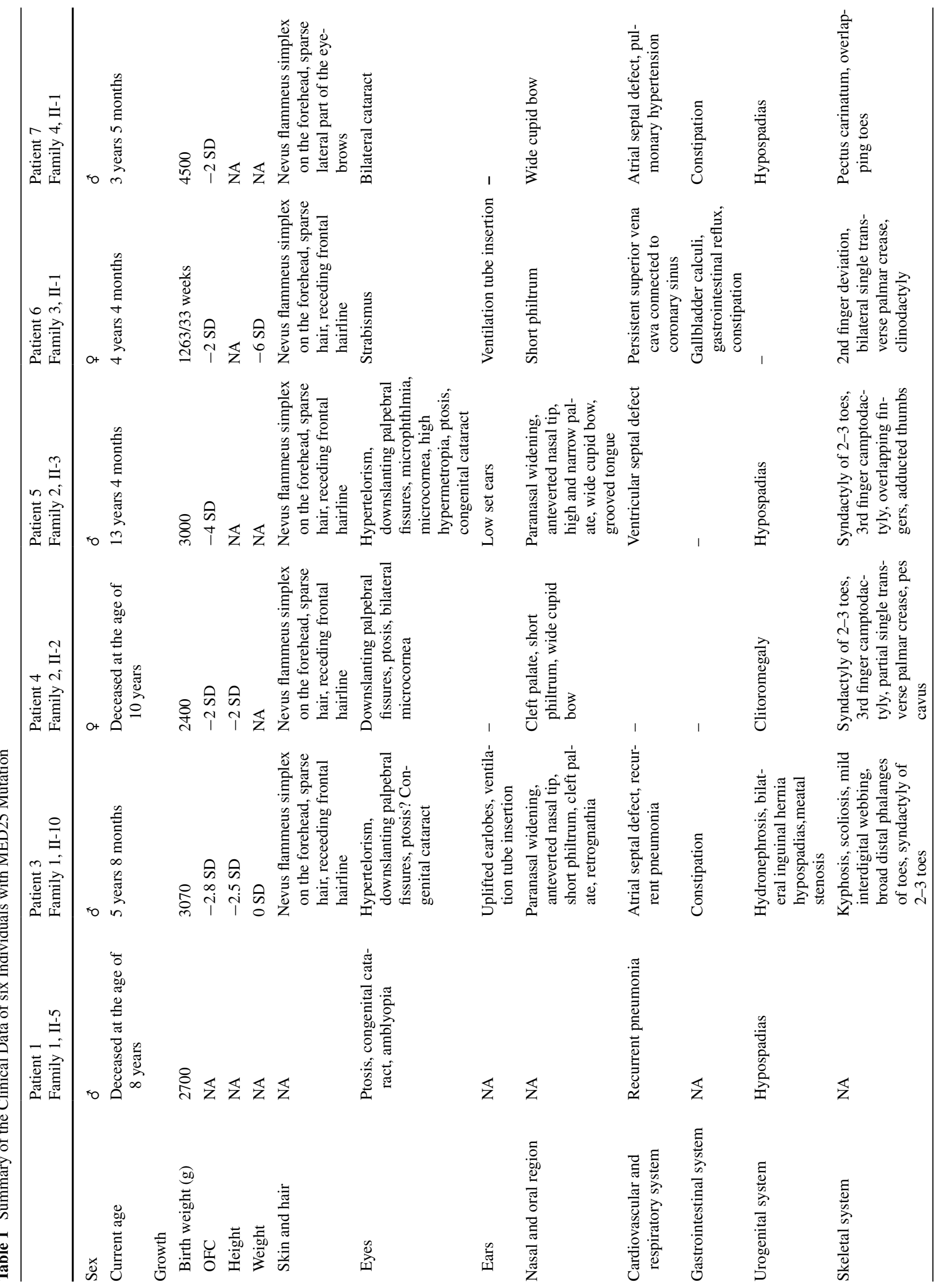




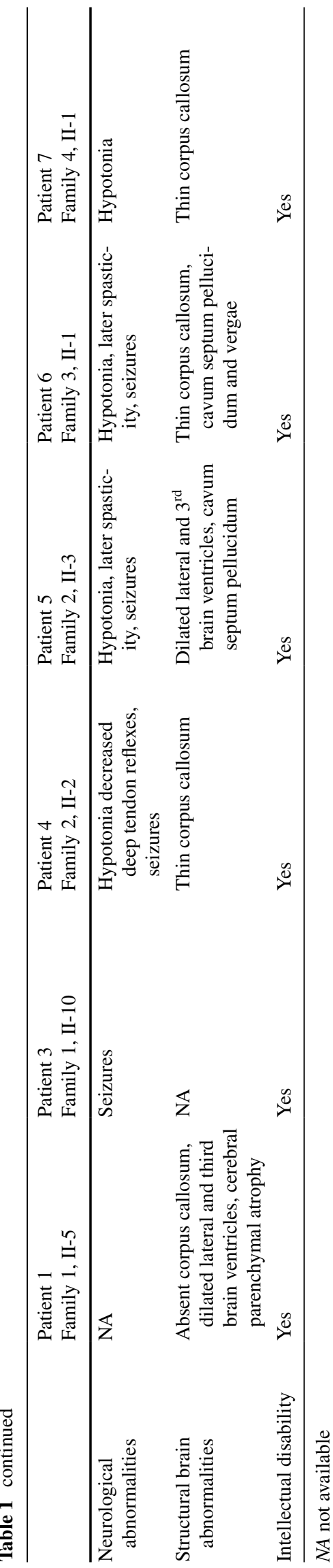

A

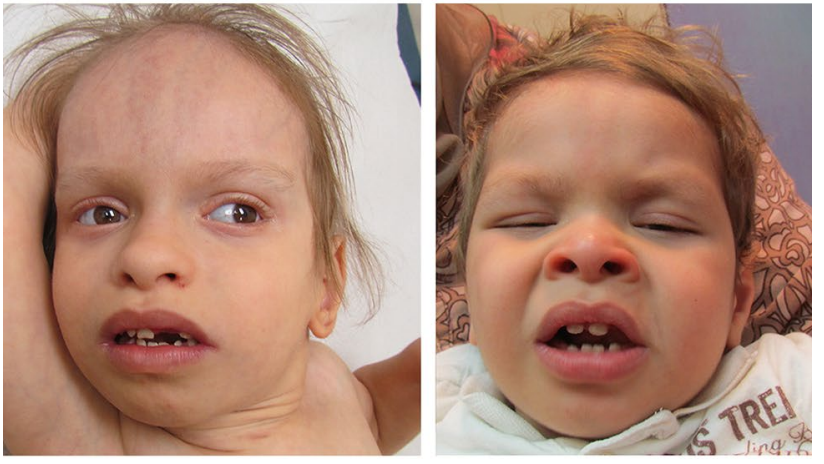

B
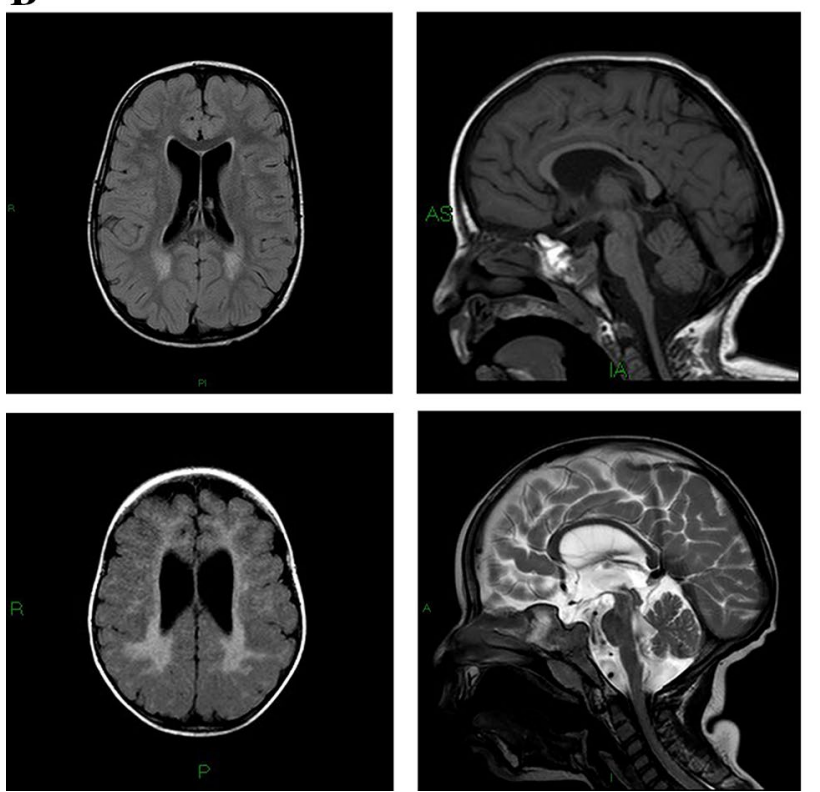

Fig. 1 Facial features of individuals with MED25 mutations. a Patient 6 (Family 3, II-1) at the age of 3 years (left) and patient 7 (Family 4, II-1) at the age of 2 years (right). Facial dysmorphic features include nevus flammeus simplex on the forehead, receding frontal hairline, sparse hair, sparse eyebrows, transparent skin, epicanthal folds, hypertelorism, short philtrum, wide cupid bow, tented upper lip and everted lower lip vermilion. b Brain MRI images of the affected individuals. Upper row, patient 3 (Family 1, II-10): axial FLAIR image at the age of 2 years and 8 months shows abnormal hyperintense signal in the occipital periventricular white matter bilaterally (left); on midline sagittal images a thin corpus callosum is noted (right). Lower row, patient 7 (Family 4, II-1): axial FLAIR image at the age of 1 year and 2 months shows abnormal hyperintense signal in the periventricular white matter of frontal and occipital region bilaterally, but mainly in the occipital regions (left). On midline sagittal images a thin corpus callosum is noted (right)

Patient 5 (Fig. 2a, Family 2, II-3) is the third child of first-cousin parents of Israeli Arab origin (Table 1). His mother was noted to have bilateral ptosis. Individual was born after an uneventful pregnancy at 40 weeks of gestation by vaginal delivery. At 2 months of age, he suffered from pneumococcal meningitis. At the age of one year 


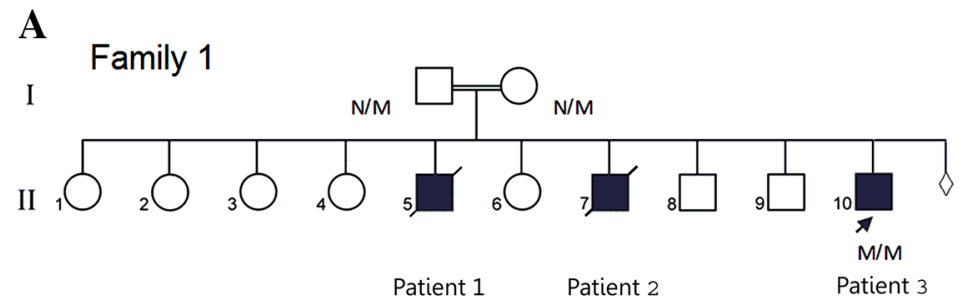

Family 2

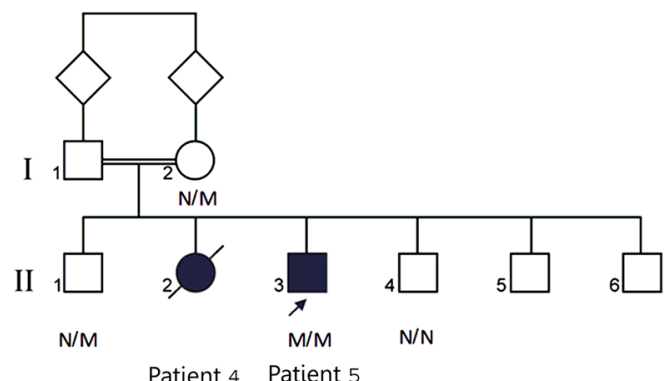

B
Family 3

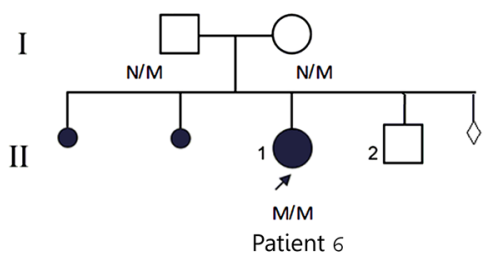

Family 4

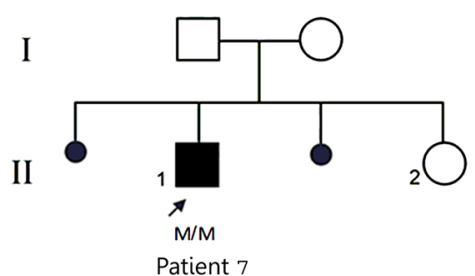

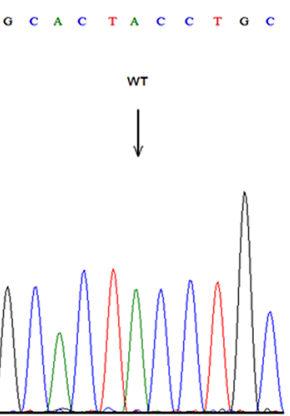

Fig. 2 Pedigrees and mutation analysis. a Pedigrees and mutation segregation in the families with affected individuals. Individuals affected or likely affected are represented by filled black symbols.
$N$ indicates the wild-type allele and $M$ indicates the mutation. b Sequence chromatograms show the homozygous mutant (M), the heterozygous carrier (C) and a non-carrier (WT) individual the patient was observed to have gross psychomotor retardation and hearing loss. He had head lag with inability to straighten his back and thus unable to carry his own weight. There was lack of movements on the right side, as well as a weak grab with his left hand. The patient was noted to have a friendly disposition and a constant smile. Clinical features, among others (Table 1) included microphthalmia, microcornea, congenital cataract and grooved tongue.

Patient 6 (Fig. 2a, Family 3, II-1) came into the clinic at 3 years presented with multiple congenital anomalies, severe microcephaly and global developmental delay. Clinical examination showed for strabismus but there were no structural eye abnormalities (Table 1). She had failure to thrive and chronic otitis media. The brainstem evoked response audiometry (BERA) examination showed hearing loss, mainly conductive, $45 \mathrm{~dB}$ on the right and $65 \mathrm{~dB}$ on the left. Visual evoked potentials (VEP) examination was normal. She could sit independently but could not stand unsupported. At the age of 4 years, she had a one-word vocabulary. Seizures were well controlled by Valproate treatment.

Patient 7 (Fig. 2a, Family 4, II-1) is the first of two children born to non-related Israeli Arab parents originating in the same village. This patient was born after a pregnancy complicated with polyhydramnios at 40 weeks of gestation by vaginal delivery. Eye examination showed bilateral cataract (additional clinical features are listed in Table 1). He had a good eye contact. At the age of 3 years, he had no words and had severe psychomotor retardation. Brain MRI images of the affected individual at the age of 1 year and 2 months showed abnormal hyperintense signal in the periventricular white matter of frontal and occipital region bilaterally, but mainly in the occipital regions (Fig. 1b).

None of the affected individuals, including a 13-yearold patient, developed ability to speak or were able to walk independently. All affected individuals have large nevus flammeus simplex on the forehead. Other features common to all or most of the patients include sparse hair, sparse eyebrows, transparent skin, epicanthal folds, hypertelorism, 
Table 2 Segregation of candidate variants identified by whole exome sequencing

\begin{tabular}{lllllll}
\hline Gene list & chr & Position & REF & ALT & Substitution list & Segregation \\
\hline NPAS1 & chr19 & 47544375 & C & T & p.(Arg212Cys) c.1208C $>$ T c.683C $>$ T & Variant did not segregate with the disease phenotype \\
MED25 & chr19 & 50321714 & A & G & p.(Tyr39Cys) & Variant segregate with the disease phenotype \\
MYH14 & $\operatorname{chr19}$ & 50735262 & G & A & p.(Arg342Gln) p.(Arg350Gln) & Variant segregate with the disease phenotype \\
ZNF525 & $\operatorname{chr19}$ & 53884101 & T & C & p.(Ile54Thr) p.(Ile90Thr) & Variant did not segregate with the disease phenotype \\
LOC284379 & $\operatorname{chr19}$ & 54103610 & G & A & p.(Thr131Ile) & Variant did not segregate with the disease phenotype \\
\hline
\end{tabular}

short philtrum, wide cupid bow, tented upper lip and everted lower lip vermilion (Fig. 1a).

Laboratory evaluation, performed in at least one affected individual, included blood cell count and peripheral blood smear, renal and liver function tests, total bilirubin, uric acid, albumin, blood electrolytes, blood amino acids, thyroid function tests, blood very-long-chain fatty acids, blood transferrin analysis to exclude congenital disorders of glycosylation (CGD), urine organic acids and urine mucopolysaccharides. All the results were normal.

\section{Whole exome sequencing}

The coverage recorded for the six obtained exome sequencing samples demonstrated an average coverage of $71 x$ $(12 \mathrm{x} \pm \mathrm{SD})$. The homozygotic genomic area (chr19: 450,000,000-550,000,000) contained 2600 targeted regions (787Kbp total sequenced) in 399 genes. None of these regions had null coverage in all samples (Supplemental List S1). The average coverage of regions per sample was $>100 \times($ Supplemental Table S2). Under a hypothesis of homozygous recessive mode of inheritance, a total of 17 variants segregating with the disease were identified. Namely, all affected individuals displayed homozygous mutations, whereas parents displayed heterozygosity for a normal and a disease allele. Interestingly, 14 of these 17 variants formed $\mathrm{a} \sim 7 \mathrm{Mb}$ homozygosity region in the two affected individuals and spanning approximately through chromosome 19: 47,500,000-54,743,000. The three additional variants were found in chromosomes 1,5 and 19. A total of twelve variants were ruled out due to their published global minor allele frequencies, to concentrate on the rare variants in the population, since this syndrome is presumed to be very rare. The remaining five variants were all missense variants found in NPAS1, MED25, MYH14, ZNF525 and LOC284379 genes (Table 2). Segregation studies, completed on all additional patients that were not included in the exome genotyping, filtered out all but two gene variants: $M Y H 14$ c.1049G>A [NM_001077186 p.(Arg350Gln) rs373908919] and MED25 c.116A>G [NM_030973 p. (Tyr39Cys)]. Although the segregation studies on the MYH14 gene have not ruled it out (Table 2), the position of its mutated amino acid was not found to be conserved (ConSurf analysis, data not shown). In addition, this variant has been found in homozygous state in two control individuals in our local database; moreover, this variant has been found many times in the general population in the ExAC database (Supplemental Table S2), excluding it from further studies. Mutations in this gene are associated with non-syndromic autosomal dominant hearing loss, while parents of all the affected individuals and all the patients had normal hearing, except for the patient 6 with the history of bacterial meningitis and patient 5 with conductive hearing loss.

DNA of 204 chromosomes from control individuals of Arab origin was tested for MED25 gene variant by restriction analysis. None of the individuals tested carried the mutation found in the affected individuals. Screening for MED25 gene variant c.116A $>$ G [p.(Tyr39Cys), NM_030973] in random 80 healthy individuals was performed in the same Muslim Israeli Arab village from where the families with MED25 mutation originated. Among the village founders were families whose ancestors migrated from Sudan and descendants of families who came to Palestine from Egypt accompanying Muhammad Ali's troops in 1834. Mutation testing revealed that $7 / 80$ of the subjects were carriers of p.(Tyr39Cys) mutation, thus reflecting the founder effect among the inhabitants of the village.

\section{Bioinformatics analysis}

Regions that are evolutionarily conserved between human and various species are shown in supplemental Figure S1A. The 747-residue protein includes 4 domains (Supplemental Figure S1B). The MED25 Y39C mutation is located towards the end of the coding region of exon 1 in a conserved position of the VWA domain (Fig. 3c). VWA-related domain is implicated in the recruitment of the Mediator complex. Although both tyrosine and cysteine are polar residues, replacement of the aromatic amino acid tyrosine by any other residue is not tolerated and is predicted to be probably damaging with high score (0.97, PolyPhen-2) (Adzhubei et al. 2010). According to the NCBI HomoloGene database, high conservation among vertebrates is found for MED25 protein, with $99 \%$ identity between human and rhesus, $91 \%$ identity with mouse, $93 \%$ identity 

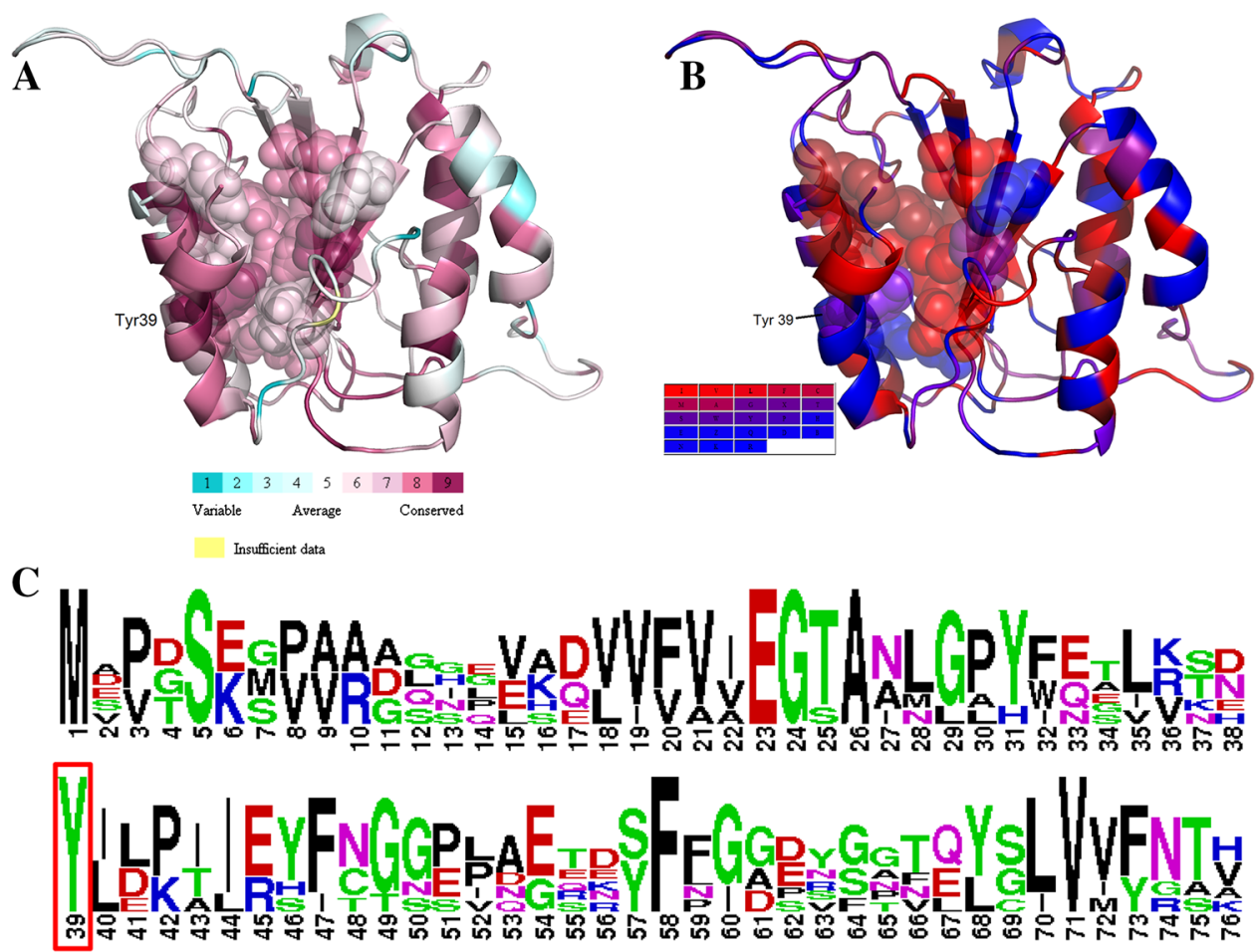

Fig. 3 MED25 protein structure prediction. a MED25 model structure presented as ribbons and colored by conservation scores as calculated by Consurf, based on the multiple sequence alignment described in "Materials and methods". The most conserved residues are colored burgundy and the most variable residues are colored cyan. Residues in the hydrophobic core are presented as transparent spheres. The figure was generated using Pymol. b MED25 model structure presented as ribbons and colored by the hydrophobicity scale of Kyte-Doolittle (hydrophobic residues in red, hydrophilic

with rat and $79 \%$ with zebrafish. The protein is also found in invertebrates and in plants. MED25 is ubiquitously expressed; with the highest levels present in the brain, heart, kidney, peripheral leukocytes, placenta, skeletal muscle and spleen (Yang et al. 2004 and UniProt). The model structure of human MED25 suggests that Tyr39 is located on an alpha-helix, pointing towards the core of the domain, surrounded by conserved hydrophobic residues from adjacent helix and beta strands (Fig. 3). A cysteine in position 39 may compromise the hydrophobic core formation during domain folding or may change the disulfide bonding network in this domain and thus disrupt the proper folding of this domain.

\section{Functional consequences of the Y39C mutation}

The Y39C point mutation is localized in the MED25 region (VWA domain) responsible of the MED25 recruitment into the Mediator complex (Mittler et al. 2003). To detect potential effects of this mutation on MED25-Mediator interaction, co-immunoprecipitation experiments were performed residues in blue). Residues in the hydrophobic core are presented as transparent spheres. The figure was generated using Pymol. c Sequence logo view of the first 70 residues of MED25 (Y39 position is highlighted in red rectangle) from Domain Mapping of Disease Mutations (DMDM) database. Amino acids are colored according to their chemical properties: polar residues in green, basic in blue, acidic in red, and hydrophobic in black. The overall height of the letter within the stack reflects the relative frequency of the residue at this position

upon transfection in mammalian cells (as described in the "Materials and methods") (Verger et al. 2013). When WTMED25 was recovered with anti-Flag antibodies, subunits encompassing all Mediator modules were also pulled down. By contrast, none of these Mediator subunits was recovered when YC-mutated MED25 was immunoprecipitated. Our results clearly indicate that the YC point mutation in the MED25 VWA domain dramatically impairs MED25 interaction with the Mediator complex (Fig. 4).

\section{Discussion}

In this study, we show that a homozygous mutation in $M E D 25$ is responsible for a hitherto previously undescribed syndrome characterized by severe intellectual disability, characteristic dysmorphic features, congenital eye abnormalities, corpus callosum abnormalities and other congenital abnormalities. While this manuscript was under revision, an article was published on homozygous missense p.(Arg140Trp) mutation in MED25 in a large 


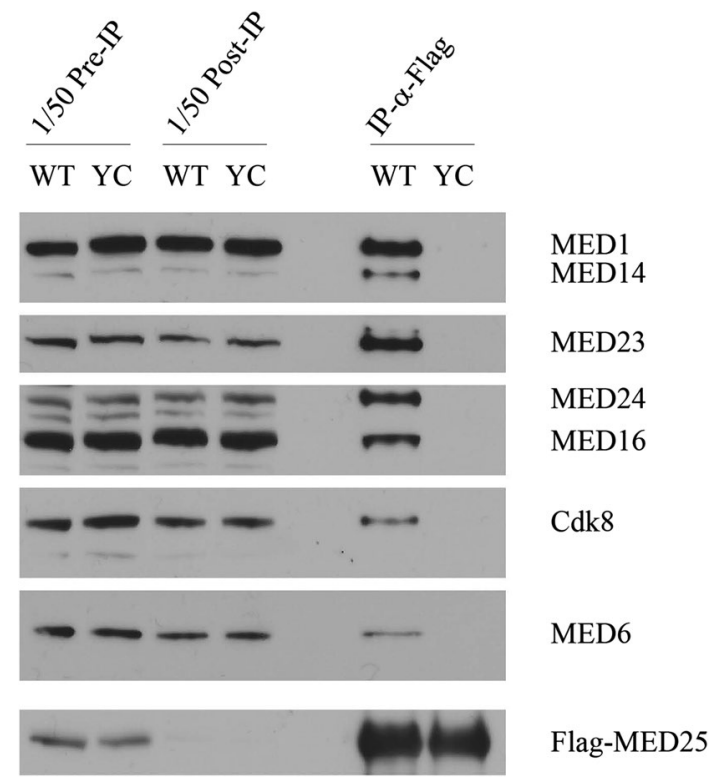

Fig. 4 The Y39C MED25 mutation impaired Mediator recruitment. WT or YC mutated Flag-NLS-MED25 was expressed in 293-HEK cells. Cellular extracts were precipitated with anti-Flag antibody (IP- $\alpha$-Flag) and various co-immunoprecipitated Mediator subunits (Med1, Med6, Med14, Med16, Med23, Med24 and CdK8) were detected with the corresponding antibodies. Aliquots of the same extracts (before and after immunoprecipitation) were analyzed with the same antibodies to detect exogenous proteins in the cellular extracts. Anti-Flag detection was also performed to assess precipitation efficiency and comparable WT and YC MED25 production levels

consanguineous family from Northeastern Brazil in which seven adults were diagnosed with syndromic intellectual disability (Figueiredo et al. 2015). Facial features described in the present study, such as prognathism, prominent chin and very large and overhanging nose tip, were not observed in the affected individuals described in that article, possibly due to the evolvement of these features with age. Eye abnormalities were not described in the reported patients, while in the families described in our report cataract and, in some cases, microcornea were present in 6 out of 7 affected individuals. A thin corpus callosum was also a constant feature amongst our patients. The differences observed between the patients reported by Figueiredo et al. and the patients described in this report might be attributable to the different localization and severity of the mutation, although both mutations are localized within VWA domain.

The clinical and radiological features observed in the patients described in this study partially overlap with other eye-brain syndromes such as MICRO syndrome and eye-muscle-brain syndromes; however, the overall constellation of features is recognizable and distinct from other syndromes. Mutation in MED25 was associated with axonal form of Charcot-Marie-Tooth disease type 2B2 in one study (Leal et al. 2009). However, no additional cases have been described since the original publication in 2009 and further evidence is needed to prove that mutations in MED25 cause CMT2B2.

We identified a carrier rate of $8.7 \%$ among the inhabitants of the village from which all the families described in this study originated. The high prevalence of the mutation can be explained both by the founder effect owing to the generally high consanguinity rate among the inhabitants of the village, and also because several families in this village had excessive numbers of intellectually disabled offspring due to founder mutations of other genes related to intellectual disability (CC2D1A gene) and thus were unacceptable as marriage partners to the other families in the same village (Basel-Vanagaite et al. 2006b).

In eukaryotes, protein-coding genes are transcribed by RNA polymerase II. The initiation of transcription and its regulation requires initiation factors, DNA-binding transcription factors as well as transcription co-activators. The Mediator complex is the complex that forms the bridge between transcription factors bound at the upstream regulatory elements and the general transcription machinery at the core promoter region (Kornberg 2005). It functions as a transcriptional co-regulator by conveying signals from activators and repressors to the RNA polymerase II transcription machinery (Malik and Roeder 2010). It has approximately thirty polypeptide subunits that are highly conserved from human to yeast; the complex has four parts including a core, a head, a tail, and a kinase module. The MED25 subunit is found exclusively in higher eukaryotes. MED25 is believed to function in chromatin modification and pre-initiation complex assembly by recruiting CBP and the Mediator complex (Lee et al. 2007). It regulates the activation of transcription by several transcription factors, including the retinoic acid receptor RAR $\alpha$ (Lee et al. 2007), the orphan receptor $\mathrm{HNF} 4 \alpha$ (Rana et al. 2011) and the chondrogenic factor Sox9 (Nakamura et al. 2011). Downregulation of MED25 by siRNA specifically reduces RAR but not thyroid hormone receptor activity, and stimulation of RAR by MED25 has been correlated with enhanced RA cytotoxicity in vivo (Lee et al. 2007). Depletion of MED25 disrupts the association of several PEA3 group transcription factors with the Mediator complex in vitro (Verger et al. 2013). We demonstrated that the Y39C point mutation in the MED25 VWA domain dramatically impairs MED25 interaction with the Mediator complex, thus strongly suggesting that this mutation is causative of the disease. Interestingly, in zebrafish, morpholino-mediated knockdown of MED25 leads to palatal malformation (Nakamura et al. 2011), resembling the palatal phenotype observed in our patients. Other genes encoding MED complex proteins that are known to be involved in the pathogenesis of human disorders include autosomal recessive intellectual disability (MED23), microcephaly, postnatal progressive, with 
seizures and brain atrophy syndrome (MED17), X-linked Ohdo syndrome, Lujan-Fryns syndrome and Opitz-Kaveggia syndrome (MED12). These syndromes, as the syndrome described here, are all characterized by intellectual disability. It was shown using two-hybrid in vitro assays that the interactions of mutated Med11 subunit with Med17, Med22, and Rad3 were decreased (Esnault et al. 2008).

In summary, we have described seven patients with a new clinically recognizable Eye-ID syndrome and have shed light on the pleiotropic clinical effects of the MED25 mutation.

\section{Web resources}

The URLs for data presented herein are as follows:

UniProt (Universal Protein Resource), http://www.uniprot.org/uniprot/Q71SY5.

Online Mendelian Inheritance in Man (OMIM), http:// www.omim.org/.

About MODELLER, https://salilab.org/modeller/about_ modeller.html.

Pymol, http://www.pymol.org/.

Acknowledgments The authors thank the families for their cooperation. This study was supported by Israeli Ministry of Health Chief Scientist foundation [No 3-4963] and Israeli Science Foundation [No 558/09]. Additionally, we would like to thank the Humanitarian Genetic Counselling Fund and Rambam Medical Center for partial support of this study. We thank the Nord-Pas de Calais Regional Council (Project Emergent) for supporting this study. The authors declare that they have no conflict of interest.

\section{References}

Adzhubei IA, Schmidt S, Peshkin L et al (2010) A method and server for predicting damaging missense mutations. Nat Methods 7:248-249. doi:10.1038/nmeth0410-248

Basel-Vanagaite L, Attia R, Yahav M et al (2006a) The CC2D1A, a member of a new gene family with $\mathrm{C} 2$ domains, is involved in autosomal recessive non-syndromic mental retardation. $\mathrm{J}$ Med Genet 43:203-210. doi:10.1136/jmg.2005.035709

Basel-Vanagaite L, Taub E, Halpern GJ et al (2006b) Genetic screening for autosomal recessive nonsyndromic mental retardation in an isolated population in Israel. Eur J Hum Genet 15:250-253. doi:10.1038/sj.ejhg. 5201750

Benkert P, Tosatto SCE, Schomburg D (2008) QMEAN: a comprehensive scoring function for model quality assessment. Proteins Struct Funct Bioinform 71:261-277. doi:10.1002/prot.21715

Benkert P, Kunzli M, Schwede T (2009) QMEAN server for protein model quality estimation. Nucleic Acids Res 37:W510-W514. doi:10.1093/nar/gkp322

Esnault C, Ghavi-Helm Y, Brun S et al (2008) Mediator-dependent recruitment of TFIIH modules in preinitiation complex. Mol Cell 31:337-346. doi:10.1016/j.molcel.2008.06.021

Figueiredo T, Melo US, Pessoa ALS et al (2015) Homozygous missense mutation in MED25 segregates with syndromic intellectual disability in a large consanguineous family. J Med Genet 52:123-127. doi:10.1136/jmedgenet-2014-102793
Finn RD, Clements J, Eddy SR (2011) HMMER web server: interactive sequence similarity searching. Nucleic Acids Res 39:W29W37. doi:10.1093/nar/gkr367

Finn RD, Bateman A, Clements J et al (2014) Pfam: the protein families database. Nucleic Acids Res 42:D222-D230. doi:10.1093/ nar/gkt1223

Hunter S, Jones P, Mitchell A et al (2012) InterPro in 2011: new developments in the family and domain prediction database. Nucleic Acids Res 40:D306-D312. doi:10.1093/nar/gkr948

Jaber L, Bailey-Wilson JE, Haj-Yehia M et al (1994) Consanguineous matings in an Israeli-Arab community. Arch Pediatr Adolesc Med 148:412-415

Jaber L, Halpern GJ, Shohat T (2000) Trends in the frequencies of consanguineous marriages in the Israeli Arab community. Clin Genet 58:106-110. doi:10.1034/j.1399-0004.2000.580203.x

Jaroszewski L, Rychlewski L, Li Z et al (2005) FFAS03: a server for profile-profile sequence alignments. Nucleic Acids Res 33:W284-W288. doi:10.1093/nar/gki418

Katoh K, Standley DM (2013) MAFFT multiple sequence alignment software version 7: improvements in performance and usability. Mol Biol Evol 30:772-780. doi:10.1093/molbev/mst010

Kornberg RD (2005) Mediator and the mechanism of transcriptional activation. Trends Biochem Sci 30:235-239. doi:10.1016/j. tibs.2005.03.011

Kumar P, Henikoff S, Ng PC (2009) Predicting the effects of coding non-synonymous variants on protein function using the SIFT algorithm. Nat Protoc 4:1073-1081. doi:10.1038/nprot.2009.86

Kyte J, Doolittle RF (1982) A simple method for displaying the hydropathic character of a protein. J Mol Biol 157:105-132. doi:10.1016/0022-2836(82)90515-0

Landau M, Mayrose I, Rosenberg Y et al (2005) ConSurf 2005: the projection of evolutionary conservation scores of residues on protein structures. Nucleic Acids Res 33:W299-W302. doi:10.1093/nar/gki370

Leal A, Huehne K, Bauer F et al (2009) Identification of the variant Ala335Val of MED25 as responsible for CMT2B2: molecular data, functional studies of the SH3 recognition motif and correlation between wild-type MED25 and PMP22 RNA levels in CMT1A animal models. Neurogenetics 10:275-287. doi:10.1007/s10048-009-0183-3

Lee H-K, Park U-H, Kim E-J, Um S-J (2007) MED25 is distinct from TRAP220/MED1 in cooperating with CBP for retinoid receptor activation. EMBO J 26:3545-3557. doi:10.1038/ sj.emboj. 7601797

Malik S, Roeder RG (2010) The metazoan Mediator co-activator complex as an integrative hub for transcriptional regulation. Nat Rev Genet 11:761-772. doi:10.1038/nrg2901

McGuffin LJ, Roche DB (2010) Rapid model quality assessment for protein structure predictions using the comparison of multiple models without structural alignments. Bioinformatics 26:182188. doi:10.1093/bioinformatics/btp629

Mittler G, Stühler T, Santolin L et al (2003) A novel docking site on Mediator is critical for activation by VP16 in mammalian cells. EMBO J 22:6494-6504. doi:10.1093/emboj/cdg619

Nakamura Y, Yamamoto K, He X et al (2011) Wwp2 is essential for palatogenesis mediated by the interaction between Sox 9 and mediator subunit 25. Nat Commun 2:251. doi:10.1038/ncomms 1242

Rana R, Surapureddi S, Kam W et al (2011) Med25 is required for rna polymerase II recruitment to specific promoters, thus regulating xenobiotic and lipid metabolism in human liver. Mol Cell Biol 31:466-481. doi:10.1128/MCB.00847-10

Šali A, Potterton L, Yuan F et al (1995) Evaluation of comparative protein modeling by MODELLER. Proteins Struct Funct Bioinform 23:318-326. doi:10.1002/prot.340230306

UniProt (2014) Activities at the universal protein resource (UniProt). Nucleic Acids Res 42:D191-D198. doi:10.1093/nar/gkt1140 
Verger A, Baert J-L, Verreman K et al (2013) The Mediator complex subunit MED25 is targeted by the N-terminal transactivation domain of the PEA3 group members. Nucleic Acids Res 41:4847-4859. doi:10.1093/nar/gkt199

Vorup-Jensen T, Ostermeier C, Shimaoka M et al (2003) Structure and allosteric regulation of the alpha $\mathrm{X}$ beta2 integrin I domain. Proc Natl Acad Sci 100:1873-1878. doi:10.1073/pnas.0237387100

Vriend G (1990) WHAT IF: a molecular modeling and drug design program. J Mol Graph 8:52-56. doi:10.1016/0263-7855(90)80070-V

Wiederstein M, Sippl MJ (2007) ProSA-web: interactive web service for the recognition of errors in three-dimensional structures of proteins. Nucleic Acids Res 35:W407-W410. doi:10.1093/nar/ gkm290

Wilson D, Pethica R, Zhou Y et al (2009) SUPERFAMILY-sophisticated comparative genomics, data mining, visualization and phylogeny. Nucleic Acids Res 37:D380-D386. doi:10.1093/nar/ gkn762

Yang F, DeBeaumont R, Zhou S, Naar AM (2004) The activatorrecruited cofactor/Mediator coactivator subunit ARC92 is a functionally important target of the VP16 transcriptional activator. Proc Natl Acad Sci 101:2339-2344. doi:10.1073/ pnas.0308676100 